\title{
VMT MIX MODELING FOR MOBILE SOURCE EMISSIONS FORECASTING: FORMULATION AND EMPIRICAL APPLICATION
}

Chandra R. Bhat and Harikesh S. Nair

University of Texas at A ustin

Department of Civil Engineering

ECJ 6.806, Austin, Texas 78712

Tel: (512) 471-4535, Fax: (512) 475-8744

E-mail addresses: bhat@mail.utexas,edu, harikesh@mail.utexas.edu

\section{ABSTRACT}

This paper proposes and implements a fractional split model that predicts the VMT mix on links as a function of the functional roadway classification of the link, the physical attributes of the link, the operating conditions on the link, and the attributes of the traffic analysis zone in which the link lies. The fractional split model is an useful formulation for VMT mix analysis because it accommodates boundary values of fractional V M T in a vehicle class, is easy to estimate using commonly available econometric software, and is easy to apply in forecasting mode to predict the VM T mix on each link of a network. The empirical analysis in the paper applies the fraction split model structure to estimate a VMT mix model for the Dallas-Fort Worth metropolitan region in Texas. Results of model evaluation are also presented.

Keywords: VMT mix, mobile-source emissions modeling, air quality analysis, fractional split model, emissions factors. 


\section{BACKGROUND AND SIGNIFICANCE OF WORK}

The integration of transportation planning and air quality planning is important for mobile source emissions estimation. The Environmental Protection A gency (EPA) requires the use of M OBILE 5 for such emissions estimation for all areas except California which uses the EM FAC 7F model.

The emissions factor models (MOBILE5 or EMFAC7F) require several traffic-related inputs, including travel speeds, vehicles miles of travel, on-road operating conditions (operating mode of vehicles, environmental conditions, existence of inspection/maintenance programs, etc. ), vehicle age distribution by vehicle class in the area of analysis, and vehicle mileage accumulation rates by vehicle class. These are used to calculate emissions factors (in grams per mile of vehicle travel for each pollutant) for eight different vehicle classes. The vehicle-class specific emissions factors are then applied to the V M T accumulated by each of the eight vehicle classes, and finally aggregated across all vehicle classes to obtain total vehicular emissions.

The level of detail at which the emissions analysis is conducted varies quite substantially among metropolitan regions. But the EPA requires that metropolitan planning areas rated as serious or higher in non-attainment designation for ozone and carbon-monoxide estimate their mobile source emissions using network-based transportation models. The planning organizations in these areas, in general, conduct their emissions analysis at an individual link level. This involves the estimation of volumes and speeds on each network link in the metropolitan area from travel demand models, followed by the computation of link-specific emissions based on a) link VMT, b) vehicle speed on the link, c) the vehicle class-specific emissions factors, and d) VMT mix fractions in the eight vehicle classes. Of these, the link VMT and link speeds are obtained 
directly from the network-based travel demand models. The vehicle class-specific emissions factors are obtained from the emissions factor models based on the various inputs listed earlier. The VMT fraction by vehicle class (referred to as VMT mix in the MOBILE5 model) is a supplementary traffic-related parameter that is to be provided by the analyst.

The emissions factors for each of the three pollutants; carbon monoxide (CO), V olatile organic compounds ( $\mathrm{OOC}$ ), and oxides of nitrogen ( $\mathrm{NOx}$ ); vary quite widely among the different vehicle classes. Consequently, the emissions analysis is very sensitive to V M T mix. For example, at high temperatures, a $2.8 \%$ change in the heavy duty gas vehicle (HDGV) mix causes about a $10 \%$ change in the CO emissions rate, and a $4.8 \%$ change in the HDGV mix leads to about a $10 \%$ shift in the VOC emissions rate (see Chatterjee et al. [1], page 45). It is, therefore, important to provide accurate VMT mix values at the individual link level (see NCHRP Research Results Digest [2], which identifies improvement in V M T mix modeling as an area of research that would be particularly beneficial for emissions modeling). The purpose of the current paper is to propose and implement a methodology for obtaining improved link-specific VMT mix values compared to those obtained from extant methods. Specifically, we develop a fractional split model that predicts the V M T mix on links as a function of the functional roadway classification of the link, the physical attributes of the link, the operating conditions on the link, and the attributes of the traffic analysis zone in which the link lies.

The rest of this paper is structured as follows. The next section reviews the state of the art/practice in VMT mix modeling. Section 3 presents the structure of, and the estimation technique for, the fractional split model used in the current paper. Section 4 provides an overview of the data sources used in the study and discusses issues related to data preparation. Section 5 
presents empirical results. Section 6 focuses on model application. Section 7 discusses model evaluation issues. The final section concludes the paper.

\section{STATE OF THE ART/PRACTICE IN VMT MIX DETERMINATION}

The emission factor models require the VM T split by eight vehicle classes. The vehicle classes are based on the size and weight of vehicles as well as the type of fuel used. The eight vehicle classes are: light-duty gasoline vehicle (LDGV), light-duty gasoline truck, type 1 (LDGT 1), light-duty gasoline truck, type 2 (LDGV2), heavy duty gasoline vehicle (HDGV), light duty diesel vehicle (LDDV), light duty diesel truck (LDDT), heavy duty diesel vehicle (HDDV), and motorcycle (MC).

The current practice in many metropolitan areas is to accept the aggregate default V M T mix computed by MOBILE5 and to apply this mix to all network links. The default VM T mix is based on national data reflecting the proportion of travel by each vehicle type in urban areas.

A nother approach adopted by some metropolitan agencies is to use 24-hour local vehicle classification-counts (rather than M OBILE5 default values) to determine V M T mix, followed by the application of factors to convert vehicle types in traffic counts to the eight M OBILE5 vehicle classes. EPA recommends that local agencies adopt this approach because the M OBILE5 default values may not be reflective of the local traffic vehicle mix. In this local vehicle count-based approach, the VMT mix is typically stratified by the functional classification of roadways to accommodate variations across roadway classes. However, since most counts are conducted only on higher roadway classes (such as interstates and major arterials), there is inadequate information to comprehensively capture variations in VMT mix by roadway class. Values of VMT mix 
obtained for the higher roadway classes are applied (sometimes after ad hoc adjustments based on judgement) to the lower roadway classes (such as minor arterials, collectors, and local roads).

A problem with the state of the art/practice discussed above for VMT mix determination is that they apply aggregate-level values across links in the road network in a region. In an analysis of VMT mix from 477 different count sites in the U.S., Chatterjee et al. [1] find substantial variation in VMT mix across the sites, emphasizing the need for local determination of VMT mix values (rather than using M OBILE default values). The same study also indicates substantial variation in VMT mix even after controlling for roadway class at any given site, underscoring the need to consider explanatory factors other than roadway class in local VM T mix analysis.

The discussion above motivates the research in this paper. Specifically, we formulate and estimate a fractional split model that determines the VMT mix ratio as a function of several informative variables, including the physical attributes of links (such as number of lanes and whether the link is a divided road or not), the operating characteristics of links (such as link speed), aggregate area type characterizations of the traffic survey zone in which the link lies (such as urban, suburban, and rural), and the land use attributes of the zone (such as retail acreage in zone and manufacturing/warehouse acreage in zone). Such a model will enable accurate V M T mix computations at a fine level of geographic resolution. 
Bhat and Nair

\section{FRACTIONAL SPLIT MODEL STRUCTURE}

\subsection{G eneral Background}

Fractional response dependent variables arise naturally in many transportation and other analysis contexts. Examples of such variables include the proportion of freight tonnage for a commodity group moving from an origin to each of several destinations, the proportion of intercity trips made by each of several travel modes, time spent by an individual in one of several activity types (shopping, social-recreational, personal business, etc.), and (as in the present analysis) the fraction of VMT accrued by each vehicle class. A characteristic of all these analyses is that the variable of interest is in the form of fractions. The sum of the fractions across all categories of the variable is equal to one, and each fraction is bounded between zero and one. Further, one or more of the fractions may take the boundary values of zero or one. In the subsequent discussion, we present the fractional split model structure in the context of VM T mix analysis.

$M$ athematically, let $y_{q i}$ be the fraction of VMT accrued by vehicle type $i(i=1,2, \ldots, l)$ on link $q$. Let this fraction be a function of a vector $x_{q}$ of relevant explanatory variables associated with attributes of the link and the traffic analysis zone in which the link lies. A common approach to analyzing fractional dependent variables is to model the log-odds ratio as a linear function (for example, see Bhat and M isra [3]):

$$
E\left(\log \frac{y_{q i}}{y_{q 1}}\right)=\beta_{i}^{\prime} x_{q}, i \neq 1,
$$

where $\beta_{\mathrm{i}}$ is a parameter vector to be estimated for each i (except a base category which needs to be normalized to zero for identification; in the above equation, the first category is arbitrarily 
considered as the base category; i.e., $\beta_{1}=\mathbf{0}$, where $\mathbf{0}$ is the null vector of the appropriate size). $\mathrm{y}_{\mathrm{q} 1}$ is the VMT fraction accrued by the first vehicle type. If some parameters in the $\beta_{\mathrm{i}}$ vectors are equal across categories, such restrictions can be imposed by jointly estimating all $\beta_{i}$ vectors after appropriate data structuring (see Bhat and M isra, [3]).

The specification in equation (1) is attractive since the transformed dependent variable in the regression is unbounded and can take values anywhere on the real line as $\mathrm{y}_{\mathrm{qi}}$ varies between 0 and 1. Thus, a linear regression is appropriate. However, as pointed out by Papke and W ooldridge [4], the specification has at least two major problems. First, the dependent variable is undefined when the fraction of VM T in a vehicle class is zero or one. If the number of vehicle class-observation combinations for which the boundary conditions prevail are few, arbitrary small adjustments may be made before computing the log-odds ratio without significantly affecting the estimated parameters. However, if there are several vehicle class-observation combinations for which the boundary conditions prevail, the adjustments can have a substantial impact on estimation. In our analysis, the fractional VM T for some vehicle types (such as buses and trucks) is zero for a large percentage of observations (i.e., links) for which vehicle classification counts are available. ${ }^{1} \mathrm{~A}$ second problem with the specification in equation (1) is that, even if the econometric specification in equation (1) is appropriate and well-defined, one cannot

${ }^{1}$ If the dependent variable represents proportions from a fixed number of groups with known group sizes, suitable adjustments have been proposed in the econometric literature (see M addala [5]; page 30). However, the corresponding Berkson's minimum chi-squared estimation method is not applicable when the fractions arise naturally in analysis settings (as in the current V M T mix setting) rather than arising as a result of the discrete grouping of disaggregate observations. 
obtain $E\left(y_{q i} \mid x_{q}\right)$ (which is of primary interest for VMT fraction forecasting) without additional assumptions about the distribution of the residuals, $u_{q i}=\log \left[y_{q i} / y_{q 1}\right]-\beta_{i}^{\prime} x_{q}(i=2,3, \ldots l)$. If a distribution is assumed or estimated, then $E\left(y_{q i} \mid x_{q}\right)$ may be computed by first obtaining the conditional (on residuals) expected value for each fraction and then unconditioning out the residuals by integrating over the distribution of the assumed or estimated distribution for the residuals (see Bhat [6] for an application of this method). However, this approach is either nonrobust (if an incorrect parametric distribution is assumed) or cumbersome (if a non-parametric distribution for the residuals is estimated). A lso, the integration in this method will involve as many dimensions as the number of vehicle types, and this can become tedious.

\subsection{Q uasi-L ikelihood E stimation}

The model we propose here for V M T mix modeling is a polychotomous extension of the binary fractional split model proposed by Papke and W ooldridge [4]. The approach does not need any ad hoc adjustment for boundary values of the dependent variable fractions and directly specifies a model for $E\left(y_{q i} \mid x_{q}\right)$. At the same time, the approach is easy to implement and is robust since we make no assumptions about the distribution of $y_{\mathrm{qi}}$ conditional on $\mathrm{x}_{\mathrm{q}}$. The focus is on consistent estimation of the parameters appearing in the conditional mean specification $E\left(y_{\mathrm{qi}} \mid \mathrm{x}_{\mathrm{q}}\right)$ and on consistent, asymptotically robust, estimation of the standard errors of the conditional mean parameters.

Consider the following econometric specification:

$$
E\left(y_{q i} \mid x_{q}\right)=G_{i}\left(\beta, x_{q}\right), 0<G_{i}(.)<1, \sum_{j=1}^{1} G_{j}(.)=1 \text {, where } \beta=\left(\beta_{2}^{\prime}, \beta_{3}^{\prime}, \ldots, \beta_{1}^{\prime}\right)^{\prime} \text {. }
$$


$G_{i}() \quad.(i=1,2, \ldots l)$ in the above equation is a pre-determined function and the properties specified above for it ensure that the predicted fractional V M T in each vehicle class for any link will lie in the interval $(0,1)$ and will sum to 1 across vehicle classes. The econometric model in equation (2) is well-defined even if $y_{q i}$ takes on the value of 0 or 1 with positive probability. The reader will note that the specification above does not make any assumption about the true underlying conditional distribution of $\mathrm{y}_{\mathrm{qi}}$ given $\mathrm{x}_{\mathrm{q}}$. This is considered unknown and can have any underlying structure.

The $\beta$ parameter vector in the conditional mean model of equation (2) is estimated by maximizing a likelihood function associated with a family of probability distributions which does not necessarily contain the true unknown distribution. The label "quasi-likelihood estimation" is used for such estimations (see Gourieroux et al. [7]). Specifically, we use the multinomial loglikelihood function in the quasi-estimation:

$$
\mathscr{L}_{q}(\beta)=\sum_{i=1}^{1} y_{q i} \log G_{i}\left(\beta, x_{q}\right) .
$$

The multinomial quasi-likelihood estimator used above belongs more generally to the linear exponential family (LEF). Gourieroux et al. [7] prove the strong consistency and asymptotic normality of the parameter estimator of the conditional mean (i.e., the elements of the $\beta$ vector) obtained by maximizing $\sum_{q} \mathscr{L}_{q}(\beta)$, as long as (and if and only if) $\mathscr{L}_{q}(\beta)$ belongs to the LEF (see also Wooldridge [8]). This is a very strong result, since it is based only on the correct specification of the conditional mean function of equation (2). The result holds irrespective of the true distribution of $\mathrm{y}_{\mathrm{qi}}$ conditional on $\mathrm{x}_{\mathrm{q}}$. Of course, if we are able to specify correctly this true distribution, we can maximize the true likelihood function to obtain a more efficient estimator than 
the quasi-likelihood estimator used here. However, the disadvantage of this alternative approach (compared to the quasi-approach) is that the resulting "true-likelihood" estimator is inconsistent under an incorrect assumption for the true distribution.

W ithin the family of LEF-based quasi-likelihood estimators, asymptotic efficiency can be achieved if the functional form of the true conditional second order moment (i.e., variance) of $\mathrm{y}_{\mathrm{qi}}$ given $\mathrm{x}_{\mathrm{q}}$ is known. This is unlikely to be the case. We prefer to base our inference only on the conditional mean specification of equation (2) and propose consistent and asymptotically robust inference for the conditional mean parameter vector $\beta$. As indicated by Papke and Wooldridge [4], this can be achieved by computing the asymptotic variance-covariance matrix of $\beta$ as $\mathrm{H}^{-1} \Delta \mathrm{H}^{-1}$, where $\mathrm{H}$ is the Hessian and $\Delta$ is the cross-product matrix of the gradients $(\mathrm{H}$ and $\Delta$ are evaluated at the estimated parameter values).

A final model structure issue concerns the specification of the functional form for $G_{i}$ in the conditional mean specification of equation (2). We use a multinomial logit functional form for $G_{i}$ since this structure is easy to program and implement. In this structure, we write:

$$
G_{i}\left(\beta, x_{q}\right)=\frac{e^{\beta_{i}^{\prime} x_{q}}}{\sum_{j=1}^{l} e^{\beta_{j}^{\prime} x_{q}}} \text {, where } \beta=\left(\beta_{2}^{\prime}, \beta_{3}^{\prime}, \ldots, \beta_{l}^{\prime}\right)^{\prime} \text {. }
$$

\section{DATA PREPARATION}

\subsection{Data Sources}

Several data sources are used in the current analysis. These include the following: a) V ehicle classification counts conducted in the Dallas-Fort W orth area by the Texas Department of Transportation's (TxDOT) Regional Planning Organization (R.P.O) and the Division 10 of 
TXD 0T, b) 1996 GIS-based road network file for the D allas-F ort W orth area, c) Zonal level land use characteristics file of the Dallas-F ort W orth area, and d) 1996 GIS-based D allas-F ort W orth zonal coverage file. The latter three data files were obtained from the $\mathrm{N}$ orth $\mathrm{C}$ entral Texas $\mathrm{C}$ ouncil of Governments (NCTCOG). Each of the four data sources are briefly discussed next.

The TXDOT vehicle classification counts used in the analysis were conducted at several fixed stations in the Dallas-F ort Worth area during the periods 1977-1987 and 1983-1993. The counts covered all the functional roadway classes, and a mixture of land-uses, with an intent to obtain a sample which is representative of the V M T mix in the region. The counts were recorded using a manual count board from 6 am to $10 \mathrm{pm}$ on weekdays in the same month every year. The 16-hour counts were expanded to a 24-hour period to obtain the 24-hour vehicle classification counts. These 24-hour counts form the basis for computing the VM T mix on links. The counts distinguished among the following vehicle types: automobiles, pickups and vans (PUV), sports utility vehicles (SUV), combination trucks ( 2 axles, 3 axles, 4 axles and 6 axles), buses ( 2 axles and 3 axles) and Motorcycles (including all two-wheelers). The counts separate trucks by the number of axles, but we combined them for the current analysis because of the very frequent occurrence of zero counts in several axle categories. We also combined 2 axle and 3 axle buses into a single bus category for the same reason. The fraction of counts of each vehicle type represent the VM T mix at the individual link level and is the dependent variable in the current analysis.

The 1996 GIS road network file includes information on the characteristics of each link in the D allas F ort W orth M etropolitan planning area. The M etropolitan A rea (M A) includes about a 4,980 square-mile area with over 45,000 unique roadway links to represent the roadway 
network. The MA covers the existing urbanized area and the contiguous area expected to be urbanized by the year 2020. It includes all of Collin, Dallas, Denton, Rockwall, and Tarrant Counties and portions of Ellis, Johnson, Kaufman, and Parker Counties. The link attributes available in the network file include length of the link, traffic direction, functional classification, number of lanes, free speed, capacity, and whether the link is divided or not.

The zonal level land use characteristics file of the $D$ allas-F ort W orth area contains land use data at the level of the traffic survey zone used by NCTCOG for their travel demand modeling purposes. There are about 6000 traffic survey zones in the Dallas Fort Worth metropolitan planning area. The land use data for each zone includes total land area, and acreage in several individual land use purposes (such as in manufacturing and warehousing, in retail, hotel and motel, in institutional buildings like churches, government, museums, schools and hospitals, and in airport runways/terminals).

The 1996 GIS-based Dallas-Fort Worth zonal coverage file provides the spatial configuration of the traffic survey zones in the Dallas F ort W orth planning area.

\subsection{Data Assembly}

The objective of the data assembly steps was to append the appropriate link/zone characteristics to each link vehicle classification count observation. To accomplish this, we first spatially overlaid the 1996 GIS road network file and the 1996 GIS zonal coverage file. Next, each link at which vehicle counts were recorded was manually queried in the network database using the name of the street and the names of the cross streets at the end nodes. Once the link at which a count was made was spatially located in the GIS road network coverage, its identifier 
number in the network file was extracted. A lso, the traffic analysis zone which spatially contains the link of interest was identified from the GIS zonal coverage. Using these link and zonal identifier fields, the relevant link and land use characteristics were mapped to each vehicle count observation.

The raw TXDOT vehicle classification counts included 370 observations of link vehicle counts, of which only 244 observations could be geo-coded in the manner discussed above. These 244 link count observations constituted the final sample for analysis. The vehicle type distribution in this final sample was almost the same as the vehicle type distribution in the raw data. ${ }^{2}$

\section{EMPIRICAL ANALYSIS}

\subsection{Sample Description}

The descriptive statistics of the dependent variable (i.e., the fractional splits among the six vehicle types across observations) in the sample is provided in Table 1. A s expected, on average, the automobile fractional split is highest, followed by the fraction of pick-ups and vans (PUV). The average percentage of sports utility vehicles (SUV) and trucks is between 3-5\%. However, at an individual link level, the SUV percentage is as high as $48.7 \%$ and the truck percentage is as high as $26.3 \%$. The fraction of buses and motorcycles in the vehicle stream is relatively low.

\footnotetext{
${ }^{2}$ The reader will note that even if the aggregate sample VM T mix does not reflect the actual aggregate VM T mix in a region, the estimated model parameters will still be consistent except for the categoryspecific constants. This is because of the multinomial logit structure of equation (4). Consistent values of the category-specific constants can be obtained in a straight-forward fashion if the aggregate vehicle type distribution in the local region is known (see Ben-A kiva and Lerman [9], page 237).
} 
The percentage of observations for which the fractional mix of trucks, buses, and motorcycles are at or very close to the boundary value of zero is rather high. In particular, the truck fraction is less than 0.01 for $33 \%$ of observations, the bus fraction is less than 0.01 for $99 \%$ of observations, and the motorcycle fraction is less than 0.01 for $95 \%$ of observations. Thus, using the log-odds analysis method (equation 1) would be inappropriate in the current modeling context. The specification in equation (2) which can accommodate boundary values of the dependent variable is the appropriate approach.

Five sets of independent variables were included in the model to predict the VM T mix on links. These are: a) link functional classification, b) link physical attributes, c) link free speed variables, d) degree of urbanization of zone (in which link lies), and e) zonal land use characteristics. A number of variables within each of the five variable classes were considered in the model specification. The final set of variables and their method of inclusion in the VM T mix model was determined based on a systematic process of eliminating variables found to be statistically insignificant in previous specifications and based on considerations of parsimony in representation. In the description below, we briefly highlight some of the characteristics of the variables in each of the five sets of variables that were retained in the final model specification.

The link functional classification identifies each link with one of five roadway classes: freeways, major arterials, minor arterials, collectors, and local/residential roads. Since the number of observations on local/residential roads were very few (only 4 out of the sample size of 244), we combined the collector and local/residential road classes into a single "collector/local" category. The sample split among the four resulting roadway classes is as follows: freeways (41.8\%), major arterials (26.2\%), minor arterials (13.5\%) and collector/local links (18.5\%). 
T wo link physical attributes turned out to be important determinants of link V M T mix: the number of lanes and whether or not the link is divided. A majority of links $(56.6 \%)$ in the sample have 2 lanes. 10.2\% of links have one lane, $24.2 \%$ have three lanes, and $9 \%$ have four lanes. A substantial percentage (82.4\%) of links are divided roads.

The link free speed varies between $9 \mathrm{mph}$ to $68 \mathrm{mph}$, with a mean value of $45 \mathrm{mph}$. A direct specification with free speed as the independent variable was inferior to the specification that categorized links into one of four free speed groups: low speed (less than or equal to $30 \mathrm{mph}$ ), low to medium speed (31-40 mph), medium speed (41-55 mph) and high speed (greater than 55 $\mathrm{mph})$.

The degree of urbanization of the zone (in which the link lies) is characterized by classifying the zone as a central business district, an urban residential area or a suburb/rural area (the differentiation between suburb and rural areas did not impact V M T mix and hence these two categories were combined into a single category). In the sample, about $5 \%$ of links are in a CBD area, about $40 \%$ in an urban area, and the remainder in a suburban/rural area.

The zonal land use variables include a) an airport presence variable indicating the presence (or absence) of airport runway/terminal facilities in the zone in which the link lies, b) an institution presence variable indicating the presence/absence of institutions such as churches, schools, and hospitals, c) zone acreage in retail and office space, and d) acreage in manufacturing plants and warehousing. In the sample, about $5 \%$ of links lie in a zone with airport-related infrastructure and about $49 \%$ of links are in zones with some land use in institutional facilities. The average zone acreage in retail/office space in the sample is 18.43 acres and the average zone acreage in manufacturing/warehousing is 31 acres. 


\subsection{Fractional Split Model Results}

The final model specification results are provided in Table 2. The table provides estimates of the $\beta$ parameter vector in equation (4).

The link functional classification variables are introduced with the freeway class being the base roadway category. The results indicate an increase in the pick-up/van (PUV) and motorcycle fractions on major and minor arterials (relative to freeways and relative to other vehicle types). The fraction of these two vehicle types, however, is highest on collector/local streets. The bus fraction is lower on minor arterials (compared to freeways and major arterials) and even lower on collector/residential streets.

The results of the effect of link physical attributes indicate an increase in truck fraction, and a decrease in bus fraction, on divided roads. M otorcycles are also more prevalent on divided highways than other non-truck vehicle types. The impact of number of lanes on vehicle mix suggests a decrease in the truck and bus fractions in the vehicle fleet on links with several lanes.

The link free speed variable coefficients show fewer PUV s and SUV s (as a fraction of total vehicles) on low speed links relative to buses/passenger cars and relative to medium/high speed links. The same, though more tempered, negative trend exists for PUV S and SUV s on low-tomedium speed links. Generally speaking, PUV s and SUV s are more prevalent on higher speed links than on lower speed links. The same is true for the truck mix in the vehicle fleet, except that this effect is much stronger than for SUV s and PUVs. The results also indicate that the bus fraction is highest on low speed facilities, and higher on low-to-medium speed facilities than on high free speed links. Finally, the fraction of motorcycles and other two wheelers is higher on medium speed links than on other links. 
The coefficients on the variables characterizing degree of urbanization show a lower fraction of trucks on links in central business district $(C B D)$ and urban residential zones relative to links in suburban/rural zones. A mong the non-truck vehicle types, the PUV fraction is likely to be lesser than the other vehicle types on CBD links and the SUV fraction is likely to be lesser than other vehicle types on urban links. A Iso, the bus fraction is highest on CBD links compared to other link types.

The final set of variables are the land use variables. The result reveal that the proportion of PUV s is high on links in zones with airport facilities. This is quite reasonable because PUVs are more convenient to transport baggage and passengers to airports. The auto proportion is high on links in zones where institutions such as churches, schools, and hospitals are present. Similarly, the auto proportion is high on links in zones with large areas allocated to retail and office space. Finally, vehicle types other than automobiles and motor cycles are likely to capture a high proportion of the VMT mix in zones with large areas invested in manufacturing plants and warehouses.

\section{MODEL APPLICATION}

The model results in Table 2 can be applied in forecasting mode to determine the V M T mix in the six vehicle types: autos, PUVs, SUVs, trucks, buses, and motorcycles/two wheelers. The implementation is particularly straight-forward using a GIS platform. This is the method that the research team is using to determine the VMT mix on each link in the Dallas-F ort W orth M etropolitan planning area as part of an ongoing air quality-related project funded by the Texas Department of Transportation (TxD OT). 
The model-predicted VM T mix in the six vehicle types has to be converted into the eightclass EPA vehicle classification for input into the M OBILE emissions factor model. We propose an approach that combines local vehicle registration data from the Dallas-F orth W orth area with information provided in the Transportation Energy Data Book (or TEDB, [10]) for this conversion, as discussed below.

The TEDB estimates that $98.8 \%$ of passenger cars are gasoline-driven and $1.2 \%$ are dieseldriven. These splits are used to allocate the "autos" VMT mix between the LDGV (light-duty gasoline powered vehicles) and LDDV (light-duty diesel powered vehicles) EPA categories. Pick-ups and vans (PUVs, including minivans and passenger vans) and sports utility vehicles (SU V s) fall under the classification of light-duty trucks and are to be assigned among the LDGT1 (light-duty gasoline-powered trucks of gross vehicle weight less than 6000 pounds), LDGT2 (light-duty gasoline-powered trucks of gross vehicle weight between 6000 and 8500 pounds), and LDDT (light-duty diesel-powered trucks of gross vehicle weight less than 8500 pounds) EPA vehicle types. From the TEDB, we computed the gasoline to diesel split for lightduty trucks as $97.88 \%$ to $2.12 \%$ based on truck sale information until 1995 . This information is used to allocate $2.12 \%$ of the "PUV " VM T mix and the "SUV " VM T mix to the LDDT category. The remaining $97.88 \%$ of gasoline-powered PUV $s$ and SUV $s$ are allocated between the less than 6000 pounds (LDGT1) and 6000-8500 pounds (LDGT2) categories based on 1996 local vehicle registration data obtained from $T$ XD OT for the Dallas-F ort W orth region. Since the local vehicle registration data are differentiated by County, and the split of the LDGT 1 and LDGT 2 categories in the registration data are quite different across counties, we developed county-specific estimates of PUV and SUV splits in the LDGT1 and LDGT2 categories (our method, of course, assumes 
that the split of traffic in the LDGT1 and LDGT 2 categories in each county is the same as the vehicle registration split in these categories in the county; the method does not consider intercounty travel which may lead to a differential LDGT1/LDGT2 traffic split vis-a-vis the registration data split in each county) .

The 1996 local vehicle registration data for the Dallas-F ort Worth area provides the gasoline-diesel splits of combination trucks (vehicles with gross weight of over 8500 pounds) by county and this information is used directly to apportion the "combination trucks" V M T mix into the HDGV (heavy-duty gasoline vehicles) and HDDV (heavy-duty diesel vehicles) EPA categories.

A "Bus" vehicle type classification is not included in the 1996 local vehicle registration data. So, we use the TEDB-estimated $20.09 \%$ to $79.91 \%$ split of buses into the gasoline-powered and diesel-powered vehicles to allocate the "bus" VM T mix between the HDGV and HDDV EPA vehicle categories.

Finally, the model-predicted "motorcycle" VMT mix is assigned completely to the motorcycle (M C) EPA vehicle category.

Table 3 provides the final county-specific conversion factors between the six-vehicle type classification typology used in the VM T mix modeling of the current paper and the eight-vehicle type EPA classification typology.

Two points to note about the conversion factors. First, these conversion factors can be updated continually as more local information becomes available. Second, the current EPA M OBILE emissions factor model does not distinguish between PUV s and SUV s; both of these are classified under light-duty trucks. Thus, the distinction between PU V s and SUV s in our V M T mix 
model is rather academic at this point. However, new versions of the MOBILE models that distinguish between emissions of PUVs and SUVs are planned. The VMT mix model proposed here can be used to provide the disaggregate input needed by these forthcoming M OBILE models.

\section{MODEL EVALUATION}

In this section, we evaluate the ability of the proposed model to replicate the actual V M T mix on links in the sample. We also compare the predicted emissions on each link (based on the proposed VMT mix model) with the actual emissions on that link (based on the observed linkspecific V M T mix values). In addition, we compare the performance of the proposed model with that of a "default" model that uses only roadway functional classification as the controlling variable for V M T mix analysis (this is the state-of-the-practice in the D allas-F ort W orth area and in other metropolitan areas which use local VM T mix values). It will be noted that the "default" model is better than using the M OBILE5 default values because it is based on local conditions. But it is restrictive compared to the proposed model in this paper because it ignores factors other than roadway classification which may affect V M T fractions.

\subsection{VMT M ix Performance Evaluation}

F or each sample link observation, we have the actual observed V M T mix in the six vehicle types: autos, PUVs, SUVs, tucks, buses, and motorcycles. We also have the corresponding model-predicted V M T mix in these six vehicle types. The evaluation of the proximity of estimated and actual VMT mixes on links is based on three criteria: the mean absolute error (MAE), the mean percentage absolute error (M PAE) and a pseudo- $\mathrm{R}^{2}$ value. The M AE is computed for each 
vehicle type as the average (across link observations) of the absolute difference between the model-predicted and actual VMT fractions for that vehicle type. The M PAE is computed as the link-averaged absolute difference between the model-predicted and actual VMT fractions as a percentage of the actual VMT fraction. The pseudo- $R^{2}$ measure is an overall model fit measure computed as follows:

$$
R^{2}=\frac{\sum_{q} \sum_{i}\left(\hat{y}_{q i}-\bar{y}_{j}\right)^{2}}{\sum_{q} \sum_{i}\left(y_{q i}-\bar{y}_{i}\right)^{2}},
$$

where $y_{q i}$ is the actual fraction of VMT accrued by vehicle type i on link $q, \quad \hat{y}_{q i}$ is the modelpredicted fraction, and $\bar{y}_{i}$ is the area-wide average VM T (from Table 1 ) for vehicle type $i$. The denominator in equation (5) is the variation in the actual link V M T mix values around the mean area-wide V M T mix value, summed across all vehicle types and links. The numerator represents the variation explained by the model. Thus, the pseudo- $R^{2}$ measure may be viewed as the fraction of total variation in VMT mix explained by the model. The measure varies between 0 and 1 .

Table 4 provides the measures of fit (M AE, M PAE and pseudo- $\mathrm{R}^{2}$ ) for the proposed model and a "default" model that uses only roadway functional classification as the controlling variable. We do not compute an MPAE for buses and motorcycles in Table 4 because the actual VMT fractions for these vehicles are extremely small, leading to substantially high M PAE values by construction.

The MAE and MPAE results in Table 4 clearly indicate the superiority of the proposed model over the default model. The MPAE for SUV s and trucks are rather high even for the proposed model, but this is an artifact of very low VMT fractions of these two vehicle types on 
many links. The more significant observation is that there is a large improvement in the fit of the proposed model relative to the default model in these two vehicle classes, especially for trucks. M ore generally, the percentage higher error in the default model compared to the proposed model is quite substantial across all vehicle types.

The pseudo- $R^{2}$ measure of the proposed and default models in Table 4 is another indicator of the superior performance of the proposed model. The results indicate that the default model is only able to explain 3\% of the variation of link V M T fractions, while the proposed model is able to explain $44 \%$ of this variation. This result implies that roadway classification alone does not contribute much in explaining V M T mix; there are several other very important link and land-use attributes that should be considered in V M T mix analysis. This is, of course, also quite apparent from the results in Table 2.

\subsection{E missions Performance E valuation}

To evaluate the benefit of the proposed VMT mix model for emissions prediction, we applied the conversion factors developed in section 6 to convert the actual/predicted count-based VM T mix fractions into VM T fractions by the eight EPA vehicle classes. Since the total volumes and the lengths of each link in the sample are known, we then computed the actual/predicted link VM T accrued by each of the EPA vehicle classes. Finally, we applied area-wide emission factors by pollutant type and EPA vehicle class (as obtained from the MOBILE5 model by the North Central Texas Council of Government Staff for the Dallas-Fort Worth area) to calculate "actual" / predicted emissions for each pollutant type on each link. The evaluation of the ability of the proposed model and the "default" model to replicate "actual" link emissions was based on the 
same three criteria; mean absolute error (MAE), mean percentage absolute error (MPAE) and pseudo- $\mathrm{R}^{2}$; as used for VMT mix analysis.

Table 5 provides the evaluation results. The M AE results in the table indicate the average link level error in the emissions predictions (in grams), while the M PA E provides the average link level percentage error in the emissions predictions. The results again indicate that the proposed model fits the data much better than the default model. The MAE in the default model is about 24-30\% higher than from the proposed model, while the M PAE in the default model is between $50-120 \%$ higher than from the proposed model depending on the pollutant type (the M PAE in Table 5 for emissions is of an order lower than that in Table 4 for VMT mix because the magnitude of link emissions is very high compared to the VMT mix fractions; thus, a 1\% error in emissions implies a much larger absolute error compared to a 1\% error in V M T mix fractions).

The pseudo- $R^{2}$ measure from the two models again emphasizes the much superior performance of the proposed model. In summary, the improvement in VM T mix predictions by the proposed model does indeed translate to improved emissions estimation.

\section{CONCLUSIONS}

VMT mix or the distribution of vehicles by weight and fuel type is an important trafficrelated parameter in determining the composite mobile source emissions on links of a network. The emissions factors (in grams per mile of vehicle travel for each pollutant) vary quite widely among different vehicle classes and therefore the emissions analysis is very sensitive to the VMT mix. Consequently, it is important to develop methods that provide accurate VM T mix values at the individual link level. 
Current approaches to V M T mix determination apply aggregate-level values across all links in the road network based on national level traffic count statistics or apply roadway-class specific values based on local vehicle classification traffic counts. H owever, it has been documented in the literature that there is substantial variation in V M T mix across different regions and across links of the same roadway class within a region.

This paper proposes a fractional split model that relates V M T mix on links to several, more informative, explanatory variables than just roadway class. The fractional split model is a valuable formulation for VMT mix analysis because it accommodates boundary values of the fractional VM T in a vehicle class, is easy to estimate using commonly available econometric software, and is easy to apply in forecasting mode to predict the VM T mix on each link of a network. A quasilikelihood approach that provides consistent and asymptotically robust inference for the parameters in the fractional split model is used in estimation.

The empirical analysis in the paper applies the fraction split model structure to estimate a V M T mix model for the Dallas-F ort W orth metropolitan region in Texas. Several data sources are used to assemble the data needed in the estimation. This assembly requires a reasonable, though not very substantial, amount of effort. Once the data is assembled, estimation of the VMT mix model proposed here is straight-forward and so is the application of the model to predict link VM T mix values. Thus, though the current paper uses the Dallas-F ort W orth region as the study area, similar models can be estimated easily in other areas. This is particularly the case today because many metropolitan areas now have network and land-use files in a GIS format, from which the information required for the proposed model estimation can be immediately extracted. 
The empirical results for the Dallas-F ort W orth area show important differences in V M T mix based on link functional classification, link physical attributes, link speed characteristics, degree of urbanization of zone that contains the link, and land-use variable of the zone in which the link lies. Model evaluation efforts indicate that the proposed model provides much better predictions of VMT mix and emissions estimation than the default model in use by M etropolitan Planning Organizations. The proposed model is currently being embedded within a GIS platform to predict the VM T mix on all links of the Dallas Fort W orth metropolitan region.

There are two limitations of the current empirical analysis. First, variations in V M T mix across different times of the day are not captured in the model. Second, seasonal variation in V M T mix are also not incorporated in our model. The vehicle classification counts used in the current paper provided only 24 -hour counts and were conducted during the same month each year. Thus, they are inadequate for capturing temporal and seasonal variations. To accommodate these variations, more extensive vehicle classification counts at different times of the day and different seasons of the year are needed. Once such data becomes available, the fractional split model structure can be applied to capture these additional effects. It is likely that the measures of fit of the proposed model in Tables 4 and 5 will improve even more after accommodating such temporal and seasonal variations in V M T mix. 


\section{ACKNOWLEDGMENTS}

This study was funded by a Texas Department of Transportation (TxDOT) project titled "Transportation Control M easure Effectiveness Evaluation in ozone N on-A ttainment A reas". The authors would like to thank Bill Knowles, Carol Nixon, Wayne $\mathrm{Y}$ oung, and George Reeves for their valuable input throughout this research effort. A rnold Breedan of the D-10 TxD OT division provided the vehicle classification count data. Ken Cervenka, M ahmoud A hmadi, and Gustavo Baez of the N orth Central Texas Council of Governments (NCTCOG) provided, and clarified data issues related to, the road network file, zonal land-use characteristics file, and the zonal coverage file for the D allas-F ort W orth area. Ken K irkpatrick and C hristopher K laus helped clarify current NCTCOG transportation air quality procedures. Finally, the authors would al so like to thank five anonymous referees for comments that considerably improved the presentation, and strengthened the technical contribution of the paper. 


\section{REFERENCES}

1. Chatterjee, A. et al. Improving Transportation Data for Mobile Source Emission Estimates, N ational Cooperative Highway Research Program Report 394, Transportation Research Board, 1997.

2. National Cooperative Highway Research Program Research Results Digest, Development of an improved framework for the analysis of air quality and other benefits and costs of transportation control measures, Transportation Research Board, National Research Council, M arch 1998.

3. B hat, C.R. and R. M isra. Discretionary activity time allocation of individuals between inhome and out-of-home and between weekdays and weekends, Transportation, Vol. 26, N o.2, 1999, 193-209.

4. Papke, L.E. and J.M. W ooldridge. Econometric methods for fractional response variables with an application to $401(\mathrm{k})$ plan participation rates, J ournal of Applied Econometrics, 11, 1996, pp. 619-632.

5. M addala, G.S. Limited D ependent and Q ualitative Variables in E conometrics, Cambridge U niversity Press, Cambridge, 1983.

6. Bhat, C.R. Modeling the Commute activity-Travel Pattern of Workers: Formulation and Empirical Analysis, Technical Paper, Department of Civil Engineering, University of Texas at A ustin.

7. Gourieroux, C., A. Monfort, and A. Trognon. Pseudo maximum likelihood methods: theory, Econometrica, 52, 1984, pp. 681-700. 
8. Wooldridge, J.M. Specification testing and quasi-maximum likelihood estimation, J ournal of Econometrics, 48, 1991, pp. 29-55.

9. Ben-A kiva, M and S. R. L erman, Discrete Choice Analysis, M IT Press, Cambridge, 1985.

10. Davis, S.C Transportation Energy Data Handbook, prepared by the Oak Ridge $\mathrm{N}$ ational Laboratory for the Office of Transportation Technologies, U.S. Department of Energy, Edition 17, 1997 . 
Table 1: Fractional Split of Vehicle Types

\begin{tabular}{||l||c|c|c|c||}
\hline \hline Vehicle Type & M ean value & Std. dev. & M inimum & M aximum \\
\hline \hline Autos & 0.653 & 0.088 & 0.389 & 0.875 \\
\hline Pick-U ps/V ans (PUV) & 0.262 & 0.062 & 0.098 & 0.416 \\
\hline Sports U tility Vehs. (SUV) & 0.035 & 0.034 & 0.000 & 0.487 \\
\hline Trucks & 0.043 & 0.045 & 0.000 & 0.263 \\
\hline Buses & 0.002 & 0.008 & 0.000 & 0.118 \\
\hline M otorcycles (MC) & 0.005 & 0.003 & 0.000 & 0.023 \\
\hline \hline
\end{tabular}


Table 2: VMT Fractional Split Model Estimation Results

\begin{tabular}{|c|c|c|}
\hline V ariable & Parameter estimate & t-statistic \\
\hline \multirow{2}{*}{\multicolumn{3}{|c|}{$\begin{array}{l}\text { Link functional classification } \\
\text { M ajor arterials }\end{array}$}} \\
\hline & & \\
\hline PUV & 0.0934 & 2.090 \\
\hline$M C$ & 0.3595 & 4.130 \\
\hline \multicolumn{3}{|l|}{ M inor arterials } \\
\hline PUV & 0.1076 & 1.177 \\
\hline Bus & -1.0570 & -2.193 \\
\hline$M C$ & 0.3138 & 2.506 \\
\hline \multicolumn{3}{|c|}{ Collector/local streets } \\
\hline PUV & 0.2416 & 3.427 \\
\hline Bus & -1.7264 & -3.140 \\
\hline MC & 0.6679 & 4.460 \\
\hline \multicolumn{3}{|c|}{ L ink physical attributes } \\
\hline \multicolumn{3}{|l|}{ Divided road } \\
\hline Truck & 1.1389 & 3.149 \\
\hline Bus & -0.6862 & -2.328 \\
\hline MC & 0.3427 & 2.625 \\
\hline \multicolumn{3}{|c|}{$\underline{\text { N umber of lanes }}$} \\
\hline Truck & -0.1738 & -2.202 \\
\hline Bus & -0.5230 & -2.045 \\
\hline \multicolumn{3}{|c|}{ L ink free speed variables } \\
\hline \multicolumn{3}{|c|}{ Low speed } \\
\hline PUV & -0.2903 & -3.838 \\
\hline SUV & -0.7688 & -6.499 \\
\hline Truck & -1.7293 & -7.013 \\
\hline Bus & 1.0436 & 2.610 \\
\hline \multicolumn{3}{|c|}{ Low to medium speed } \\
\hline PUV & -0.1469 & -2.297 \\
\hline SUV & -0.3377 & -3.259 \\
\hline Truck & -1.8454 & -11.480 \\
\hline Bus & 0.5063 & 1.744 \\
\hline \multicolumn{3}{|l|}{ M edium speed } \\
\hline Truck & -0.4125 & -3.847 \\
\hline MC & 0.1481 & 1.829 \\
\hline
\end{tabular}




\begin{tabular}{|c|c|c|}
\hline V ariable & Parameter estimate & t-statistic \\
\hline \multicolumn{3}{|c|}{ Degree of urbanization } \\
\hline \multicolumn{3}{|c|}{ Central Business District } \\
\hline PUV & -0.2919 & -3.205 \\
\hline Truck & -1.0350 & -3.409 \\
\hline Bus & 1.7342 & 4.106 \\
\hline \multicolumn{3}{|c|}{$\underline{\text { Urban residential }}$} \\
\hline PUV & -0.0918 & -1.932 \\
\hline SUV & -0.2322 & -2.395 \\
\hline Truck & -0.5645 & -4.209 \\
\hline \multicolumn{3}{|c|}{ Zonal land-use variables } \\
\hline \multicolumn{3}{|c|}{ A irport presence } \\
\hline PUV & 0.1823 & 2.211 \\
\hline \multicolumn{3}{|c|}{ Institution presence } \\
\hline A uto & 0.1207 & 2.904 \\
\hline \multicolumn{3}{|c|}{ A creage in office/retail space } \\
\hline PUV & -0.0019 & -2.399 \\
\hline SUV & -0.0038 & -1.796 \\
\hline Truck & -0.0165 & -4.282 \\
\hline Bus & -0.0146 & -1.937 \\
\hline MC & -0.0026 & -2.169 \\
\hline \multicolumn{3}{|c|}{ A creage in manufacturing/warehousing } \\
\hline PUV & 0.0009 & 3.427 \\
\hline SUV & 0.0021 & 5.494 \\
\hline Truck & 0.0067 & 9.632 \\
\hline Bus & 0.0031 & 2.457 \\
\hline
\end{tabular}

Notes: 1) PUV - Pick-ups and vans, SUV - Sports utility vehicle, M C - M otorcycles/two-wheelers

2) The estimated constant values for each vehicle type are as follows (the auto vehicle type is the base): -0.8147 (for PUV), -2.1601 (for SUV), -2.4148 (for trucks), -4.2927 (for buses), and -5.3752 (for M C). 
Table 3: TxDOT Vehicle Count Vehicle Type to MOBILE Vehicle Type C onversion Factors

\begin{tabular}{|c|c|c|c|c|c|c|c|c|}
\hline & & & & c count & & & & \\
\hline \multirow{2}{*}{$\begin{array}{l}\text { TXD OT } \\
\text { classification }\end{array}$} & \multicolumn{8}{|c|}{ EPA M OBILE vehicle type classification } \\
\hline & LDGV & LDDV & LDGT1 & LDGT2 & LDDT & HDGV & HDDV & $\mathrm{MC}$ \\
\hline A utos & $98.8 \%$ & $1.2 \%$ & - & - & $\begin{array}{l}- \\
-\end{array}$ & 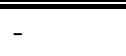 & 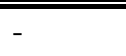 & 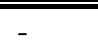 \\
\hline PUV & - & - & 95.16 & 2.72 & $2.12 \%$ & - & - & - \\
\hline SUV & - & - & 95.16 & 2.72 & $2.12 \%$ & - & & - \\
\hline Trucks & - & - & - & - & - & $35.43 \%$ & $64.57 \%$ & - \\
\hline Buses & - & - & - & - & - & $20.09 \%$ & $79.91 \%$ & - \\
\hline M otorcycles & - & - & - & - & - & - & - & $100 \%$ \\
\hline
\end{tabular}

Tarrant County

\begin{tabular}{||l||l|l|l|l|l|l|l|l||}
\hline \hline \multirow{2}{*}{$\begin{array}{l}\text { TXD OT } \\
\text { Classification }\end{array}$} & EPA M OBILE vehicle type classification \\
\cline { 2 - 9 } & LDGV & LDDV & LDGT1 & LDGT2 & LDDT & HDGV & HDDV & MC \\
\hline \hline A utos & $98.8 \%$ & $1.2 \%$ & - & - & - & - & - & - \\
PUV & - & - & $96.07 \%$ & $1.81 \%$ & $2.12 \%$ & - & - & - \\
SUV & - & - & $96.07 \%$ & $1.81 \%$ & $2.12 \%$ & - & - & - \\
Trucks & - & - & - & - & - & $39.31 \%$ & $60.69 \%$ & - \\
Buses & - & - & - & - & - & $20.09 \%$ & $79.91 \%$ & - \\
M otorcycles & - & - & - & - & - & - & - & $100 \%$ \\
\hline
\end{tabular}

Collin County

\begin{tabular}{||l||l|l|l|l|l|l|l|l||}
\hline \hline \multicolumn{1}{||l|}{$\begin{array}{l}\text { TXD OT } \\
\text { classification }\end{array}$} & EPA M OBILE vehicle type classification \\
\cline { 2 - 9 } & LDGV & LDDV & LDGT1 & LDGT2 & LDDT & HDGV & HDDV & M C \\
\hline \hline A utos & $98.8 \%$ & $1.2 \%$ & - & - & - & - & - & - \\
PUV & - & - & $96.15 \%$ & $1.73 \%$ & $2.12 \%$ & - & - & - \\
SUV & - & - & $96.15 \%$ & $1.73 \%$ & $2.12 \%$ & - & - & - \\
Trucks & - & - & - & - & - & $44.24 \%$ & $55.76 \%$ & - \\
Buses & - & - & - & - & - & $20.09 \%$ & $79.91 \%$ & - \\
M otorcycles & - & - & - & - & - & - & - & $100 \%$ \\
\hline
\end{tabular}

Denton County

\begin{tabular}{||l||l|l|l|l|l|l|l|l||}
\hline \hline \multicolumn{1}{||l|}{$\begin{array}{l}\text { TXD OT } \\
\text { classification }\end{array}$} & EPA M OBILE vehicle type classification \\
\cline { 2 - 9 } & LDGV & LDDV & LDGT1 & LDGT2 & LDDT & HDGV & HDDV & M C \\
\hline \hline A utos & $98.8 \%$ & $1.2 \%$ & - & - & - & - & - & - \\
PUV & - & - & $96.36 \%$ & $1.52 \%$ & $2.12 \%$ & - & - & - \\
SUV & - & - & $96.36 \%$ & $1.52 \%$ & $2.12 \%$ & - & - & - \\
Trucks & - & - & - & - & - & $43.30 \%$ & $56.70 \%$ & - \\
Buses & - & - & - & - & - & $20.09 \%$ & $79.91 \%$ & - \\
Motorcycles & - & - & - & - & - & - & - & $100 \%$ \\
\hline
\end{tabular}

Rockwell County

\begin{tabular}{||l||l|l|l|l|l|l|l|l||}
\hline \hline \multicolumn{1}{||l|}{$\begin{array}{l}\text { TXD OT } \\
\text { Classification }\end{array}$} & EPA M OBILE vehicle type classification \\
\cline { 2 - 9 } & LDGV & LDDV & LDGT1 & LDGT2 & LDDT & HDGV & HDDV & MC \\
\hline \hline A utos & $98.8 \%$ & $1.2 \%$ & - & - & - & - & - & - \\
PUV & - & - & $95.96 \%$ & $1.92 \%$ & $2.12 \%$ & - & - & - \\
SUV & - & - & $95.96 \%$ & $1.92 \%$ & $2.12 \%$ & - & - & - \\
Trucks & - & - & - & - & - & $34.24 \%$ & $65.76 \%$ & - \\
Buses & - & - & - & - & - & $20.09 \%$ & $79.91 \%$ & - \\
Motorcycles & - & - & - & - & - & - & - & $100 \%$ \\
\hline
\end{tabular}


Table 4: VMT M ix Performance E valuation

\begin{tabular}{|c|c|c|c|c|c|c|c|}
\hline \multirow{2}{*}{ F it Statistic } & \multirow{2}{*}{ M odel } & \multicolumn{6}{|c|}{ Vehicle Type } \\
\hline & & A uto & PUV & SUV & Trucks & Buses & Motorcycles \\
\hline \multirow{3}{*}{$\begin{array}{l}\text { M ean A bsolute } \\
\text { Error (M AE) }\end{array}$} & Proposed & 0.0482 & 0.0398 & 0.0133 & 0.0163 & 0.0016 & 0.0019 \\
\hline & Default & 0.0720 & 0.0475 & 0.0150 & 0.0350 & 0.0018 & 0.0019 \\
\hline & $\begin{array}{l}\% \text { higher error in } \\
\text { default over proposed }\end{array}$ & 49.38 & 19.35 & 12.78 & 114.72 & 12.50 & 0.00 \\
\hline \multirow{3}{*}{$\begin{array}{l}\text { M ean Percentage } \\
\text { A bsolute E rror } \\
\text { (M PAE) }\end{array}$} & Proposed & 7.60 & 17.36 & 38.45 & 44.43 & - & - \\
\hline & Default & 11.35 & 20.70 & 47.69 & 67.96 & - & - \\
\hline & $\begin{array}{l}\text { \% higher error in } \\
\text { default over proposed }\end{array}$ & 49.34 & 19.24 & 24.03 & 52.96 & - & - \\
\hline \multirow[t]{2}{*}{ Pseudo- $R^{2}$} & Proposed & \multicolumn{6}{|c|}{0.43} \\
\hline & Default & \multicolumn{6}{|c|}{0.03} \\
\hline
\end{tabular}


Table 5: E missions Performance Evaluation

\begin{tabular}{|c|c|c|c|c|}
\hline \multirow{2}{*}{ F it Statistic } & \multirow{2}{*}{ M odel } & \multicolumn{3}{|c|}{ Pollutant Type } \\
\hline & & $\begin{array}{c}\text { Carbon M onoxide } \\
(\mathrm{CO})\end{array}$ & $\begin{array}{c}\text { Volatile Organic } \\
\text { Compounds (V OCs) }\end{array}$ & $\begin{array}{c}\text { Oxides of Nitrogen } \\
(\mathrm{NOX})\end{array}$ \\
\hline \multirow{3}{*}{$\begin{array}{l}\text { M ean A bsolute } \\
\text { Error (MAE) in } \\
\text { grams }\end{array}$} & Proposed & 9992 & 684 & 6098 \\
\hline & Default & 12754 & 853 & 8030 \\
\hline & $\begin{array}{l}\text { \% higher error in } \\
\text { default over proposed }\end{array}$ & 27.64 & 24.76 & 31.70 \\
\hline \multirow{3}{*}{$\begin{array}{l}\text { M ean Percentage } \\
\text { A bsolute E rror } \\
\text { (M PAE) }\end{array}$} & Proposed & 1.84 & 1.30 & 8.71 \\
\hline & Default & 2.93 & 2.00 & 19.06 \\
\hline & $\begin{array}{l}\% \text { higher error in } \\
\text { default over proposed }\end{array}$ & 59.24 & 53.40 & 118.82 \\
\hline \multirow[t]{2}{*}{ Pseudo- $R^{2}$} & Proposed & \multicolumn{3}{|c|}{0.515} \\
\hline & D efault & \multicolumn{3}{|c|}{0.004} \\
\hline
\end{tabular}




\section{L ist of Tables}

Table 1: $\quad$ Fractional Split of V ehicle Types

Table 2: $\quad$ VMT Fractional Split M odel Estimation Results

Table 3: TXD OT V ehicle C ount V ehicle Type to M OBILE Vehicle Type Conversion F actors

Table 4: VM T M ix Performance Evaluation

Table 5: Emissions Performance Evaluation 